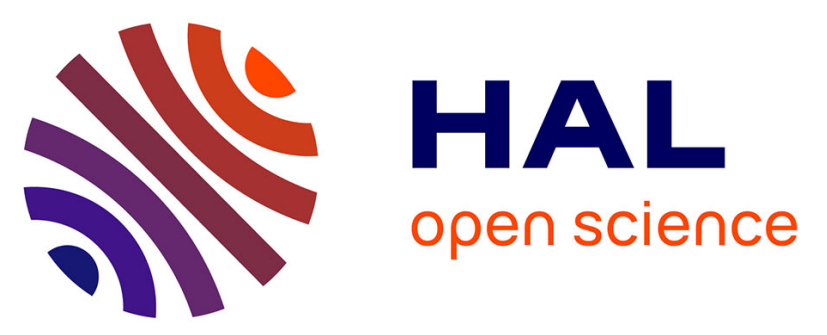

\title{
A PROBABILISTIC OBJECTIVE FUNCTION FOR 3D RIGID REGISTRATION OF INTRAOPERATIVE US AND PREOPERATIVE MR BRAIN IMAGES
}

Pierrick Coupé, Pierre Hellier, Xavier Morandi, Christian Barillot

\section{- To cite this version:}

Pierrick Coupé, Pierre Hellier, Xavier Morandi, Christian Barillot. A PROBABILISTIC OBJECTIVE FUNCTION FOR 3D RIGID REGISTRATION OF INTRAOPERATIVE US AND PREOPERATIVE MR BRAIN IMAGES. IEEE International Symposium on Biomedical Imaging: From Nano to Macro, IEEE Signal Processing Society (SPS) and the IEEE Engineering in Medicine and Biology Society (EMBS), Apr 2007, Washington DC, USA, United States. pp.1320-1323, 10.1109/ISBI.2007.357103 . inria-00147025

\section{HAL Id: inria-00147025 \\ https://hal.inria.fr/inria-00147025}

Submitted on 15 May 2007

HAL is a multi-disciplinary open access archive for the deposit and dissemination of scientific research documents, whether they are published or not. The documents may come from teaching and research institutions in France or abroad, or from public or private research centers.
L'archive ouverte pluridisciplinaire HAL, est destinée au dépôt et à la diffusion de documents scientifiques de niveau recherche, publiés ou non, émanant des établissements d'enseignement et de recherche français ou étrangers, des laboratoires publics ou privés. 


\title{
A PROBABILISTIC OBJECTIVE FUNCTION FOR 3D RIGID REGISTRATION OF INTRAOPERATIVE US AND PREOPERATIVE MR BRAIN IMAGES
}

\author{
Pierrick Coupe $e^{1,2,4}$, Pierre Hellier ${ }^{1,2,4}$, Xavier Morandi ${ }^{1,2,3,4}$ and Christian Barillot ${ }^{1,2,4}$ \\ ${ }^{1}$ University of Rennes I, UMR CNRS 6074, IRISA, F-35042 Rennes, France \\ ${ }^{2}$ INRIA, VisAGeS U746 Unit/Project, F-35042 Rennes, France \\ ${ }^{3}$ CHU, University Hospital of Rennes, rue H. Le Guillou, F-35033 Rennes, France \\ ${ }^{4}$ INSERM, VisAGeS U746 Unit/Project, F-35042 Rennes, France
}

\begin{abstract}
The registration of intraoperative Ultrasound (US) with preoperative Magnetic Resonance (MR) images is a challenging problem due to the difference of information contained in each image modality. To overcome this difficulty, we introduce a new probabilistic function for similarity measurements based on the mean curvature of MR isophots and US hyperchogenic structures. Experiments were carried out on 3 patients and compared with three other registration approaches. The results show that the proposed method converges robustly compared to the standard registration techniques, with a computational time compatible with intraoperative use.
\end{abstract}

\section{INTRODUCTION}

The information and the artifacts present in US and MR images being of different nature, the registration of these two modalities is a difficult task. Registration approaches based on classical similarity measure such as Sum Square Difference (SSD), Mutual Information (MI) or Correlation Ratio (CR) are known to fail [1]. Previous works have studied three options to register US and MR images : (a) the matching of homologous features extracted from both images [2, 3, 4, 5, 6], (b) the preprocessing of the images to make US images and MR images more similar in order to use classical similarity measures [7, 8, 9] and (c) the iconic registration based on a specific similarity measure matching the US and MR image intensities [1].

Landmark-based registration represents the majority of the approaches in the context of US to MR registration.The motivation is bound to the difficulty of finding a function matching US image intensities with MR image intensities. Based on definition of region of interest in US and MR images, these methods register points, lines or surfaces. The main disadvantage of landmark-based registration is the sensitivity to the segmentation.

Intensity-based approach using histogram-based similarity measures tends to overcome the problem by preprocessing the images in oder to register similar images. In [7] the segmentation and the magnitude gradient of the MR image are used to simulate a pseudo-US, then the Correlation Coefficient (CC) is used as the similarity measure. In [8], the Normalized Mutual Information (NMI) is used to register the gradient magnitude images of both modalities. Intensity-based approaches use the MR image gradient in the registration procedure because US images are considered as similar to gradient images.

To the best of our knowledge, Roche et al. [1] were the only to incorporate the transformation of MR image into pseudoUS in the similarity measure : the Bivariate Correlation Ratio (BCR). The registration procedure is split into two steps : (a) the estimation of the polynomial function matching the intensity and the gradient of the MRI with the US image and (b) the estimation of the transformation minimizing the BCR.

In the context of Image-Guided NeuroSurgery (IGNS), we propose a new objective function based on a probabilistic map of hyperechogenic structures composed of the mean curvature $(M l v v)$ of the preoperative MR image and the segmentation of the pathological tissues. The Mlvv operator was introduced to detect the sulci and the cerebral falx [10] which are the hyperechogenic structures of the brain in ultrasound images.

\section{METHOD}

\subsection{Probabilistic objective function}

Contrary to histogram-based approaches which match all the information in both images, the proposed approach consists in matching the informative features : the hyperechogenic structures. The registration process is based on the estimation of the transformation $\hat{T}$ maximizing the conjoint probability for a voxel $X=(x, y, z)$ to be included in hyperechogenic structure in both modalities :

$$
\hat{T}=\arg \max _{T} \int_{\Omega} p\left(X \in \Phi_{U S}, T(X) \in \Phi_{M R}\right) d X
$$

where $p\left(X \in \Phi_{U S}\right)$ is the probability for $X$ to be included in an hyperechogenic structure of the US image and $p(X \in$ 
$\left.\Phi_{M R}\right)$ is the probability for $X$ to be included in an hyperechogenic structure (in the sense of the ultrasound image) of the MR image. As the probabilities are independent, we can write :

$$
\hat{T}=\arg \max _{T} \int_{\Omega} p\left(X \in \Phi_{U S}\right) \cdot p\left(T(X) \in \Phi_{M R}\right) d X
$$

The maximization of Eq. 2 is achieved by a simplex procedure within a multiresolution scheme.

\subsection{Construction of the probability maps}

In order to construct the probability maps, we define a function $f$ matching the intensity of the US image and the MR image with the probability for $X$ to be included in an hyperechogenic structure :

$$
p(X \in \Phi)=f(u(X))
$$

where $u: \Omega \longmapsto \mathbb{R}$ is an image defined on $\Omega$. For intraoperative US image $U$, the evaluation of $f$ is done during surgery and is only a normalizing function :

$$
p\left(X \in \Phi_{U S}\right)=U(X) / 2^{n}
$$

where $n$ is the number of bits of the unsigned encoded image. For preoperative MR image $V$, the evaluation of $f$ is done prior to surgery and is based both on the Mlvv operator and the manual segmentation of the pathological tissue performed by the neurosurgeon. The Mlvv operator is used instead of gradient operator because it is more specific than the gradient operator extracting relevant features that are present in both MR and US images (see comparison of Mlvv and gradient operator Fig. 1). Thus, $f$ is defined as :

$$
p\left(X \in \Phi_{M R}\right)= \begin{cases}M \operatorname{lv} v(V(X)) / 2^{n} & \text { if } X \in M_{1} \\ \Psi(X) & \text { if } X \in M_{2} \\ 0 & \text { otherwise }\end{cases}
$$

where $M_{1}$ represents the positive value of Mlvv map computed on the brain tissue without the pathological tissue segmentation $M_{2}$. The $M l v v$ operator is defined in $3 \mathrm{D}$ as :

$$
\begin{gathered}
\operatorname{Mlvv}(V(x, y, z))=-\frac{1}{2\|\vec{w}\|^{2}} \\
\frac{\partial V(X)}{\partial x}^{2}\left(\frac{\partial^{2} V(X)}{\partial y^{2}}+\frac{\partial^{2} V(X)}{\partial z^{2}}\right)-2 \frac{\partial V(X)}{\partial y} \frac{\partial V(X)}{\partial z} \frac{\partial^{2} V(X)}{\partial y \partial z}+ \\
\frac{\partial V(X)}{\partial y}^{2}\left(\frac{\partial^{2} V(X)}{\partial x^{2}}+\frac{\partial^{2} V(X)}{\partial z^{2}}\right)-2 \frac{\partial V(X)}{\partial x} \frac{\partial V(X)}{\partial z} \frac{\partial^{2} V(X)}{\partial x \partial z}+ \\
\left.\frac{\partial V(X))^{2}}{\partial z}\left(\frac{\partial^{2} V(X)}{\partial x^{2}}+\frac{\partial^{2} V(X)}{\partial y^{2}}\right)-2 \frac{\partial V(X)}{\partial x} \frac{\partial V(X)}{\partial y} \frac{\partial^{2} V(X)}{\partial x \partial y}\right]
\end{gathered}
$$

where $\|\vec{w}\|^{2}=\frac{\partial V(X)}{\partial x}^{2}+\frac{\partial V(X)}{\partial y}^{2}+\frac{\partial V(X)}{\partial z}^{2} . \Psi(X)$ is the probability given to $X$ in the segmentation of pathological tissue $M_{2}$ and $\Psi()$ is used to incorporate a priori on pathology. For hyperechogenic pathological tissue such as cavernoma or low-grade glioma $\Psi(X)$ is high. For hyporechogenic pathological tissue such as necrosis or cyst $\Psi(X)$ is low. In other words, the Mlvv is computed from MR image on the extracted brain by masking the pathological tissue. Then, only the positive values (i.e. the sulci and the falx) are kept. Finally, the Mlvv map is fused with the manual segmentation $M_{2}$ (see Fig. 1).

\subsection{Validation framework}

Our approach was compared with three other approaches: (1) the Mutual information (MI), (2) the Normalized Mutual Information (NMI) on the gradient images of MR and US images as described in [8] and (3) the Correlation-Ratio on map extracted from MR (viewed as a "pseudo-US") and the US image. The latter can be considered similar to the method described in [7]. The simplex was used as optimization procedure within a multiresolution scheme for all the compared approaches.

\section{MATERIAL}

\subsection{Data acquisition}

$\mathrm{T} 1$ sense 3D sequences were used to acquired preoperative T1-weighted MR image on a 3T Philips Gyroscan scanner. The manual segmentation of the pathological tissue was performed before the surgery. After skull stripping from [11], the $M l v v$ was computed on the brain tissue denoised with the Non Local Means filter [12].

During the IGNS procedure, the US probe (Sonosite cranial $7-4 \mathrm{MHz}$ probe) was tracked by the Polaris cameras of the Stealth Station TREON (Medtronic Inc). The Sononav software installed on neuronavigator acquired the 2D B-scans and the probe positions. From the 2D B-scans and their positions, a 3D volume was reconstructed with the Probe Trajectory method introduced in [13].

\subsection{MR-US registration of the neuronavigation system}

At the beginning of the neurosurgical procedure, the coordinate system of the preoperative MR image and the coordinate system of the intraoperative field are related by a rigid registration. Performed by the neuronavigator, this rigid registration is based on surface matching between preoperative MR image and the position of points acquired on the patient's head with the position pointer.

\subsection{Pathology of the patients}

In this study, hyperechogenic pathologies such as cavernoma (patient 1, see Fig. 2 and patient 2, see Fig. 3) and lowgrade glioma (patient 3, see Fig. 4) have been chosen. For the experiments, we have tested the simplest model for $\Psi(X)$. According to the neurosurgeron, the lesional tissue was considered both homogenic and hyperechogenic; thus $\Psi(X)$ was 

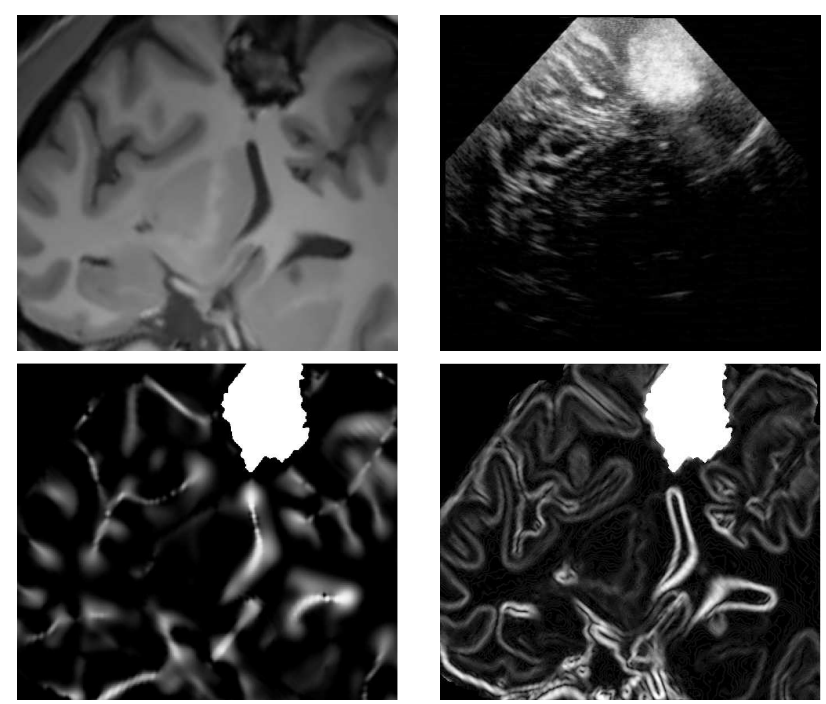

Fig. 1. Top. Left : the denoising MR images. Right : the corresponding US images obtained with the registration performed by the neuronavigation system. Bottom. Left : the probability map based on Mlvv extracted from denoising MR image. Right : the same approach with gradient magnitude extracted from denoising MR image. Visually, the Mlvv is more specific to extract relevant structure of US image from MR image.

set to 1 (see Eq. 5). Example of intraoperative image and probability map for patient 2 are presented in Fig. 1. For each patient, two sequences were acquired before opening the dura. As it is generally admitted [8], we assumed that no brainshift occurs before opening of the dura.

\section{RESULTS}

\begin{tabular}{|c|cc|cc|cc|} 
Method & \multicolumn{2}{|l|}{ Patient 1 } & \multicolumn{2}{|l|}{ Patient 2 } & Patient 3 \\
\hline MI & - & - & - & + & - & - \\
NMI on gradients & - & - & - & - & - & - \\
CR on maps & - & - & + & + & - & - \\
Our approach & + & + & + & + & + & +
\end{tabular}

Table 1. Results of the different MR/US registration methods. For each patients two B-scans sequences were studied. The registration was classified as successful (+) if visually the registration improves the overlay of the images given by neuronavigator, or unsuccessful (-) in the other cases.

Tab. 1 shows the result of the registration procedures for the different similarity measures. These results underline that MI is not adapted for this kind of multimodal registration. Our implementation of the method proposed in [8] failed on all cases, maybe due to the acoustic shadows present in the data. The correlation ratio on maps converged when the segmenta-
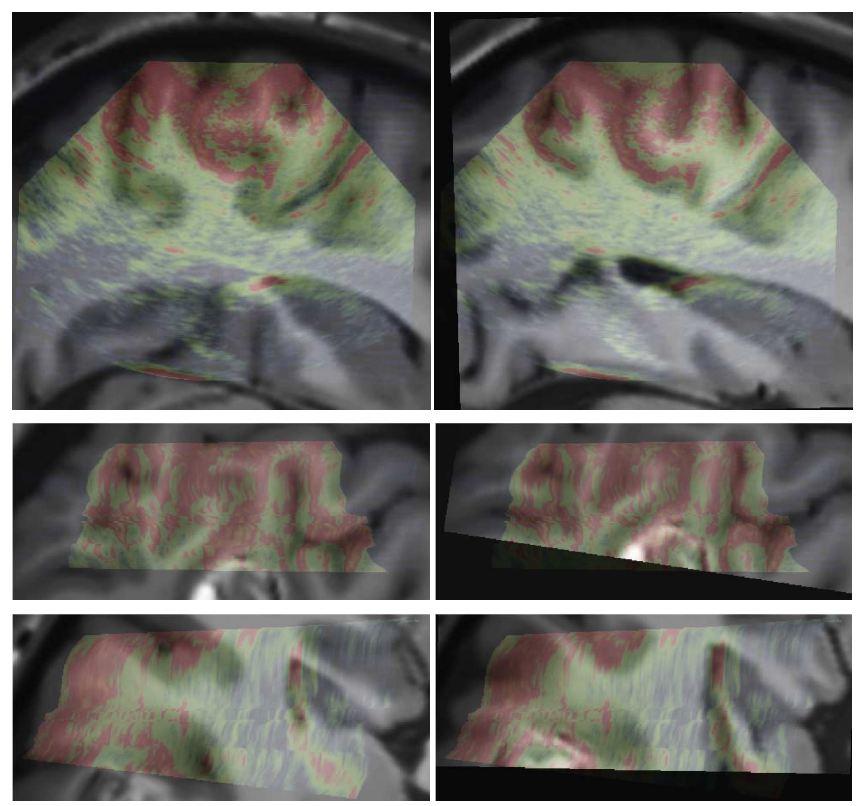

Fig. 2. Patient 1. Left : registration given by the neuronavigation system. Right : the result after correction with our registration approach. For this case, even if the lesion was not entirely included in the US volume, the proposed registration procedure converged.

tion of the pathology is entirely included in the US volume. Nonetheless, for patient 1 and 3, only a part of the lesion intersects the US volume. In these cases, this approach failed. The results obtained with our methods are presented in Fig 2, 3 and 4 . The proposed approach converged in all cases and was visually accurate. The registration procedures take less than two minutes on Pentium(R) $\mathrm{M}$ at $2 \mathrm{GHz}$. A priori defining in the preoperative MR image the most probable locations of high intensity in the intraoperative US images seems to be an efficient way to register intraoperative US and preoperative MR brain images.

\section{CONCLUSION}

This paper presents a new framework for the 3D rigid registration of US and MR brain images. This framework is based on the probabilistic objective function of the hyperechogenic brain structures extracted from MR images. The experiments performed on 3 patients show that our approach converges robustly compared to methods such as the Mutual Information (MI), the Normalized MI on the gradient images or the Correlation Ratio on the US images and the pseudoUS created from MR images. The computational burden required for our method is compatible with intraoperative use. The visually accuracy of our methods has been shown but its quantitative accuracy needs to be futher investigated. 

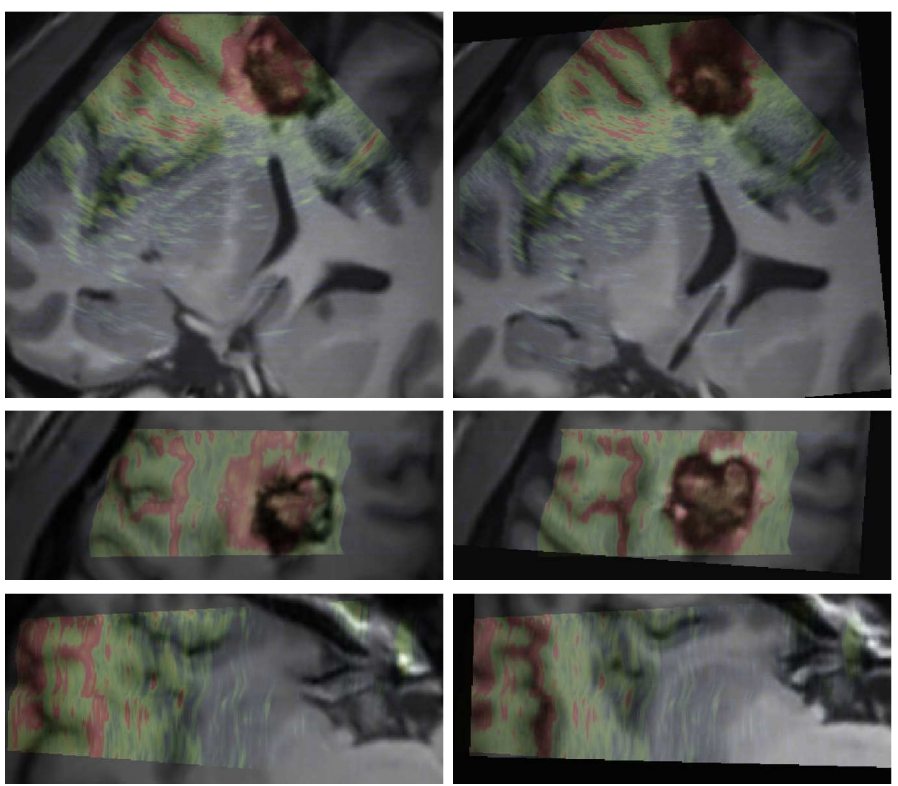

Fig. 3. Patient 2. Left : registration given by the neuronavigation system. Right : results after correction with our registration approach. In this case, the acoustic shadow artifact was present on the US image. The signal below the lesion was totally dark. The proposed approach allowed to overcome these artifacts without specific detection of the shadows.

\section{REFERENCES}

[1] A. Roche, X. Pennec, G. Malandain, and N. Ayache, "Rigid registration of 3D ultrasound with MR images : a new approach combining intensity and gradient information," IEEE TMI, vol. 20, no. 10, pp. 1038-1049, October 2001.

[2] N. Hata, M. Suzuki, T. Dohi, H. Iseki, K. Takakura, and D. Hashimoto, "Registration of ultrasound echography for intraoperative use : a newly developed multiproperty method," in SPIE, 1994, vol. 2359, pp. 251259.

[3] D. G. Gobbi, R. M. Comeau, and T. M. Peters, "Ultrasound/MRI Overlay with Image Warping for Neurosurgery," in MICCAI. 2000, vol. 1935 of LNCS, pp. 106-114, Springer.

[4] A. P. King, J. M. Blackall, G. P. Penney, P. J. Edwards, D. L. G. Hill, and D. J. Hawkes, "Bayesian estimation of intra-operative deformation for image-guided surgery using 3-D ultrasound," in MICCAI. 2000, vol. 1935 of $L N C S$, pp. 588-597, Springer.

[5] B. C. Porter, D. J. Rubens, J. G. Strang, J. Smith, S. Totterman, and K. J. Parker, "Three-dimensional registration and fusion of ultrasound and MRI using major vessels as fiducial markers," IEEE TMI, vol. 20, no. 4, pp. 354-359, April 2001.

[6] I. Reinertsen, M. Descoteaux, S. Drouin, K. Siddiqi, and D. L. Collins, "Vessel driven correction of brain shift," in MICCAI. 2004, vol. 3217 of LNCS, pp. 208-216, Springer.

[7] T. Arbel, X. Morandi, M. Comeau, and D. L. Collins, "Automatic nonlinear mri-ultrasound registration for the correction of intra-operative brain deformations," in MICCAI. 2001, vol. 2208 of LNCS, pp. 913922, Springer.

[8] M. M. J. Letteboer, M. A. Viergever, and W. J. Niessen, "Rigid registration of 3D ultrasound data of brain tumours," in CARS, 2003, pp. 433-439.
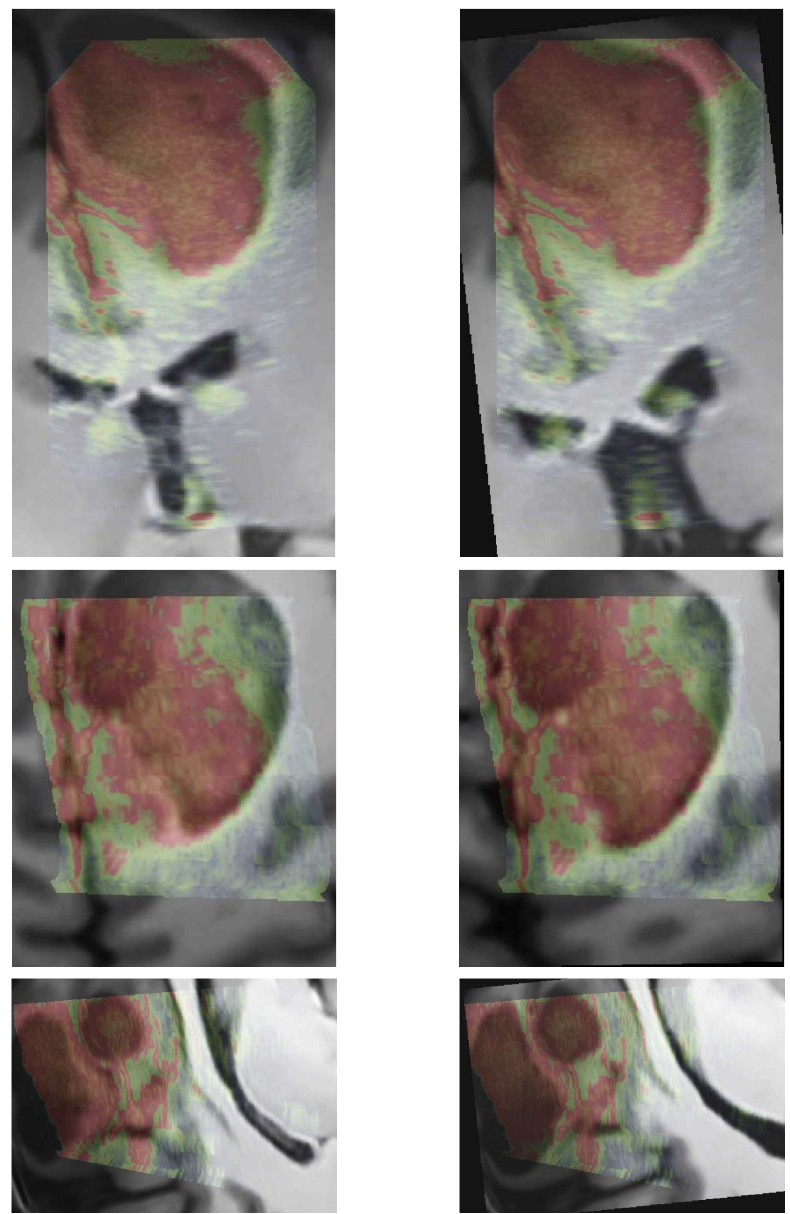

Fig. 4. Patient 3. Left : registration given by the neuronavigation system. Right : the result after correction with our registration approach.

[9] G.P. Penney, J.M. Blackall, M.S. Hamady, T. Sabharwal, A. Adam, and D.J. Hawkes, "Registration of freehand 3d ultrasound and magnetic resonance liver images," Medical Image Analysis, vol. 8, pp. 81-91, 2004.

[10] G. Le Goualher, C. Barillot, and Y. Bizais, "Three-dimensional segmentation and representation of cortical sulci using active ribbons," Int. J. of Pattern Recognition and Artificial Intelligence, vol. 11, no. 8, pp. 1295-1315, 1997.

[11] J.-F. Mangin, O. Coulon, and V. Frouin, "Robust brain segmentation using histogram scale-space analysis and mathematical morphology," in MICCAI. 1998, vol. 1496 of LNCS, pp. 1230-1241, Springer Verlag.

[12] P. Coupé, P. Yger, and C. Barillot, "Fast Non Local Means Denoising for 3D MR Images," in MICCAI. 2006, vol. 4191 of LNCS, pp. 33-40, Springer.

[13] P. Coupé, P. Hellier, N. Azzabou, and C. Barillot, "3D Freehand Ultrasound Reconstruction based on Probe Trajectory," in MICCAI. 2005, vol. 3749 of $L N C S$, pp. 597-604, Springer. 